\title{
Study of the first paramagnetic to ferromagnetic transition in as prepared samples of Mn-Fe-P-Si magnetocaloric compounds prepared by different synthesis routes
}

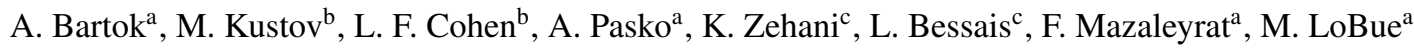 \\ ${ }^{a}$ SATIE, ENS Cachan, CNRS, Universite Paris-Saclay, 61 av President Wilson, F-94230 Cachan, France \\ ${ }^{b}$ Imperial College, London SW7 2AZ, United Kingdom \\ ${ }^{c}$ CMTR, ICMPE, CNRS-UPEC, 2-8 rue Henri Dunant, F-94320 Thiais, France
}

\begin{abstract}
Magnetocaloric materials with composition of $\mathrm{Mn}_{1.3} \mathrm{Fe}_{0.65} \mathrm{P}_{0.5} \mathrm{Si}_{0.5}$ have been prepared by ball milling and solid-state reaction methods and consolidated using powder annealing, and conventional and spark plasma sintering. Magnetic and calorimetric measurements show remarkable differences upon first cooling, and slight differences on second and further coolings between the samples prepared by different synthesis routes. Further measurements using Hall probe imaging in high magnetic field have been also carried out. As-prepared samples have been cooled down just above the critical temperature, and the first phase transition has been induced by application of a magnetic field. Bulk samples show staircase isothermal magnetization curves whereas powders show smoother transition curves.
\end{abstract}

Keywords:

Magnetocaloric effect, magnetic refrigeration, first-order magnetic transition, magnetic entropy change, $\mathrm{Fe}_{2} \mathrm{P}-\mathrm{based}$ compounds, spark plasma sintering

\section{Introduction}

Hexagonal Mn-Fe-P-Si compounds are known to represent a promising rare-earth-free road to room temperature magnetic refrigeration [1]. The giant magnetocaloric effect (MCE) has been studied in this system with particular emphasis on the tuning of the critical temperature and magnitude of the entropy change as a function of the composition [2]. Many materials where the first-order transition is associated with a strong magneto-elastic coupling show a peculiar phenomenon referred to as virgin-effect (VE) [3, 4] consisting in a lower transition temperature $T_{c}$ upon first cooling and a change in the slope of the magnetisation versus temperature curve. Our objective is to investigate the mechanism behind this strong change in behavior after the first cycle. The magnetocaloric transition in $\mathrm{Mn}-\mathrm{Fe}-\mathrm{P}-\mathrm{Si}$ alloys is extremely sensitive to intrinsic (e.g. composition, chemical disorder) as well as to extrinsic (e.g. structural disorder, strain distributions in bulk polycrystalline samples) aspects. This makes separation of these two contributions to material properties a difficult task. Here we focus our attention on the influence of the sintering technique on the virgin effect in Mn-Fe-P-Si compounds. Four samples with same nominal composition are prepared using powder annealing, conventional sintering and Spark Plasma Sintering (SPS) technique under two different compacting pressures. Isothermal and isofield measurements are carried out on the as-prepared samples near to the critical temperature. Finally, by means of Hall probe imaging and optical microscopy we show that bulk samples crack during the first paramagnetic-ferromagnetic transition. The effect of the density and stress are discussed.

\section{Experimental}

Polycrystalline samples of $\mathrm{Mn}_{1.3} \mathrm{Fe}_{0.65} \mathrm{P}_{0.5} \mathrm{Si}_{0.5}$ were prepared by high-energy ball milling (using Fritsch Pulverisette 4) under Ar atmosphere. The compounds were prepared from high purity $\mathrm{Fe}_{2} \mathrm{P}$ powder and $\mathrm{P}, \mathrm{Mn}$ and Si chips. The grinding bowl contained 198 grams of grinding balls (10 $\mathrm{mm}$ diameter) and 25 grams of the starting materials. The rotation speed was fixed at 120 rpm during the first $30 \mathrm{~min}$ (pre-milling) and then at 330 rpm for 5 hours.

The powder synthesis route we used consists in a two step annealing; the polycrystalline sample was first annealed at $1373 \mathrm{~K}$ for 2 hours in $200 \mathrm{hPa}$ Ar atmosphere, and then at $1123 \mathrm{~K}$ for 20 hours (rate of temper- 
ature increase and decrease : $1.2 \mathrm{~K} / \mathrm{min}$ ). To produce the conventionally sintered sample, the fine powder was pressed into pellets (6 mm diameter) in Ar atmosphere. Then the pellets were sealed in quartz ampules in 200 $\mathrm{hPa} \mathrm{Ar}$ atmosphere and finally the same heat treatment was used as for the annealed powder sample. The third and fourth samples were prepared using Spark Plasma Sintering (SPS) from the annealed powder sample. The polycristalline sample was heated up to $1223 \mathrm{~K}$ (using temperature changing rate of $100 \mathrm{~K} / \mathrm{min}$ ) in Ar atmosphere under 50 and $80 \mathrm{MPa}$ applied constant pressure and sintered for 30 minutes.

The use of off-stoichiometric compounds of $(\mathrm{Mn}, \mathrm{Fe})_{1.95}(\mathrm{P}, \mathrm{Si})$ instead of $(\mathrm{Mn}, \mathrm{Fe})_{2}(\mathrm{P}, \mathrm{Si})$ has been reported to be a way to reduce the presence of impurity phases and to obtain a sharp first order transition [5]. X-Ray diffraction patterns, obtained using a Bruker D8 Advance diffractometer with $\mathrm{Cu} K \alpha$ radiation, have been used to check that no secondary phases were present. Room temperature results show that the reflections can be identified with a single hexagonal $\mathrm{Fe}_{2} \mathrm{P}$-type phase $(P \overline{6} 2 m$ space group) in all cases. A small amount of cubic $(\mathrm{Mn}, \mathrm{Fe})_{3} \mathrm{Si}$ (space group $F m \overline{3} m$ ) was also detected but with a very low fraction $(<1-2 \mathrm{wt} \%)$. The lattice parameters are estimated from the XRD measurements using Rietveld refinement. According to the determined lattice parameters $(a=610 \pm 0.5 \mathrm{pm}$ and $c=344 \pm 1.5 \mathrm{pm})$ and literature data [1], all the samples are expected to be in the paramagnetic state at room temperature.

Magnetic measurements were carried out using a Lakeshore vibrating sample magnetometer (VSM) and a Quantum Design 6000 physical property measurement system (PPMS). The density was measured using the standard Archimedes method.

\section{Results}

Temperature scanning in low magnetic field is the most common way to determine the critical temperature of a material with a phase transition. Performing measurement under constant temperature change rate is a rather difficult task that can result in a lower density of points along the steepest section of the transition (as is apparent in Fig. 1 for all the bulk samples). However that is a very important prerequisite to collect reliable data, particularly when processes that irreversibly change the sample properties are present such as the VE. Here we focus on the first transition, namely the VE, of the four samples measured by changing temperature under isofield conditions $\left(\mu_{0} H=1 \mathrm{~T}\right)$, in zero field using a standard DSC equipment, and induced by the applied field in isothermal conditions, by fixing the temperature just above the Curie point of the studied phase.

\subsection{Isofield measurements}

Isofield magnetization measurements have been carried out using a VSM in a temperature range $150 \mathrm{~K}$ $\leq T \leq 320 \mathrm{~K}$. The temperature dependence of magnetization of the four samples is presented in Fig. 1.
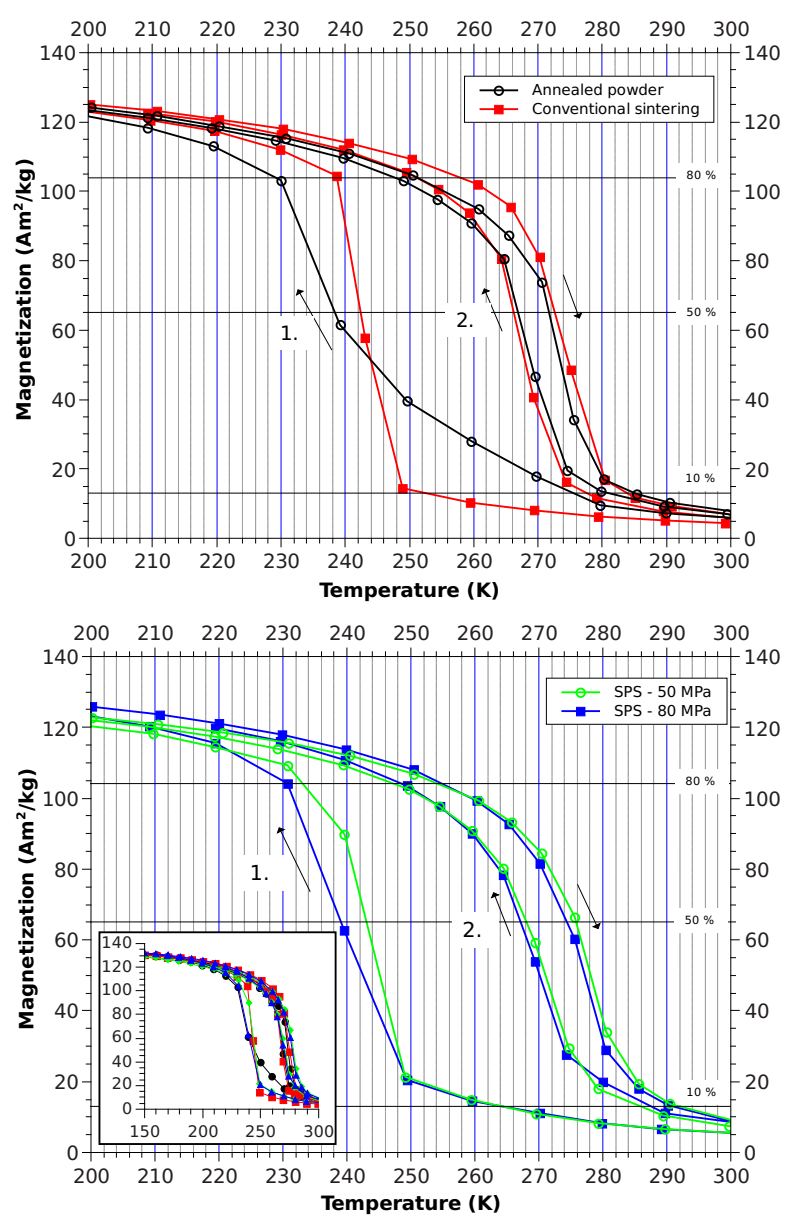

Figure 1: Temperature dependence of the magnetization, measured in $1 \mathrm{~T}$ applied field with increasing and decreasing temperature (2 cycles) on the as-prepared materials. Annealed powder sample (black hollow circles), sample prepared by conventional sintering (red full square), sample prepared by SPS under $50 \mathrm{MPa}$ (green hollow circles) and $80 \mathrm{MPa}$ (blue full square).

Looking at the second and further cooling-heating cycles, all four samples show quite sharp paramagnetic to ferromagnetic transitions at around $270 \mathrm{~K}$ (here $T_{c}$ is estimated from the steepest point in the $M(T)$ measured curves), with a thermal hysteresis of 5-7 K, and with a maximum magnetization of about $130 \mathrm{Am}^{2} / \mathrm{kg}$ at 150 $\mathrm{K}$, which is in a good agreement with the literature [5]. 
This is a further check of the absence of relevant differences of composition between the four samples.

Nevertheless differences between the behavior of the first transition curves of the four samples are apparent. To make the discussion on the virgin curves more quantitative we define a central part of the transition as the temperature interval between $10 \%$ of the maximum total magnetic moment (i.e. the magnetic moment we assume to be associated, given the applied field, with a sample that is fully transformed into the ordered phase) and the $80 \%$ of it $\left(\Delta T_{10 \%-80 \%}=T_{10 \%}-T_{80 \%}\right)$. Namely, we focus on the central section of the transition where about $70 \%$ of the sample is expected to transform from the PM phase to the FM one. This choice is mainly dictated from the need to cut out the initial and final part of the curves where magnetic moment value can be more affected by orientation processes (see for instance the discussion about the role of anisotropy in [6]). Actually, the curve where the choice of the interval would be more difficult is the smoothest one (i.e. the one relative to the powder sample) where there is no easily detectable change in slope of the curve between steep section and the initial and final tails. That is why we selected the interval after performing a temperature dependent XRD measurement of the phase fractions on the powder sample (the full results will be published elsewhere). This confirmed that, at least for the powder sample, in the chosen interval $M$ can be properly used as a measure of the amount of FM phase fraction present in the sample. Due to the sharpness of the VE transition, and to the need to control $d T / d t$, virgin curves measured on bulk samples are often made by few points in the most abrupt region of the transition. That makes it difficult to detect the steepest point to identify it with the first cooling critical temperature. Due to this difficulty in extrapolating a critical temperature associated with the virgin curve, we use the temperature corresponding to $50 \%$ of $M, T_{50 \%}$, by linearly interpolating between points on the curves, to define in some way the position of the VE. Virgin curves together with a first heating curve and a second cooling curve are compared in Fig. 1 (powder and classical sintering sample in the top frame, the two SPS samples in the bottom one, all measurement together in the inset). Values of $\Delta T_{10 \%-80 \%}$ and of $T_{50 \%}$ can be found in Table 1 .

\subsection{DSC measurements}

Further study of the virgin effect on our samples have been carried out using a PerkinElmer PYRIS Diamond differential scanning calorimeter. The measured heatflow is shown in Fig. 2 as a function of the temperature.
In the case of the stable curves (second and further coolings) the position of the maximal heat flow can be easily detected for all the samples and we have $T_{c}=269 \pm 3 \mathrm{~K}$ with a slight $T_{c}$ shift with respect to magnetization measurements due to the effect of the $1 \mathrm{~T}$ applied field in the latter. First cooling DSC signals are quite different; in the case of bulk samples they show a sawtooth shape due to the presence of individual abrupt avalanche-like events. In this case we limit ourselves to detect the temperature interval where the VE takes place, $\Delta T_{D S C}$ (summarized in Table 1), by identifying the initial and final temperature of the first cooling transition as the points where the first cooling signal departs from the second cooling one.
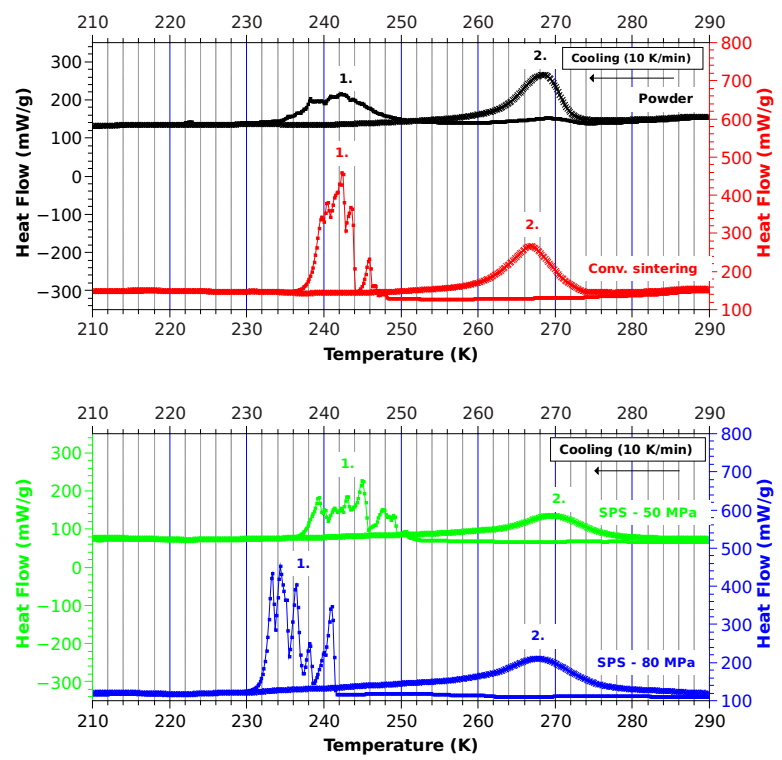

Figure 2: DSC measurements on the as-prepared samples during the first (curve 1) and second (curve 2) cooling cycle (cooling rate : 10 $\mathrm{K} / \mathrm{min}$ ).

\subsection{Field-induced transition}

The field-induced first PM-FM transition has been carried out using a PPMS magnetometer up to $5 \mathrm{~T}$ applied field. The as prepared samples have been cooled down slowly (using the No overshoot temperature settling mode) close to the critical temperature $(250 \mathrm{~K})$. The isothermal magnetization curves have been measured in increasing and deceasing field performing two full cycles. Results are presented in Fig. 3. No difference can be observed between the curves after the first field increasing, namely after the first transition has been field induced. This is indeed what we would expect from isofield measurements as, after the first 


\begin{tabular}{|c||cccccc|}
\hline Sample & $\begin{array}{c}\text { Density } \\
\left(\mathrm{g} / \mathrm{cm}^{3}\right)\end{array}$ & $\begin{array}{c}\Delta T_{10 \%-80 \%} \\
(\mathrm{~K})\end{array}$ & $\begin{array}{c}T_{50 \%} \\
(\mathrm{~K})\end{array}$ & $\begin{array}{c}\mu_{0} \Delta H_{10 \%-80 \%} \\
(\mathrm{~T})\end{array}$ & $\begin{array}{c}\mu_{0} H_{50 \%} \\
(\mathrm{~T})\end{array}$ & $\begin{array}{c}\Delta T_{D S C} \\
(\mathrm{~K})\end{array}$ \\
\hline \hline Annealed powder & - & 46.9 & 238.5 & 3.9 & 1.0 & 22 \\
Conventonal sintering & 5.76 & 13.4 & 242.4 & 1.9 & 2.0 & 12 \\
SPS-50 MPa & 6.03 & 30.4 & 243.1 & 3.8 & 2.4 & 14 \\
SPS-80 MPa & 6.11 & 32.1 & 239.1 & - & - & 11 \\
\hline
\end{tabular}

Table 1: Density and critical temperature data from isothermal and isofield magnetization measurements and DSC.

cooling, at $250 \mathrm{~K}$ all the samples are expected to be fully in the ordered phase. On the contrary, the first transition curves of the four samples are quite different. To discuss these differences we define, similarly to the isofield case, a field interval for the central part of the transition as the difference between the field where $80 \%$ and $10 \%$ of the total magnetization respectively are achieved, $\Delta H=H_{80 \%}-H_{10 \%}$ (also reported in Table 1). The field where the magnetization is the $50 \%$ of total $M, H_{50 \%}$ is also reported. The virgin field induced transition of the powder sample (black hollow circles in Fig. 3) is smooth, as we would expect in analogy with isofield results (both, $M(T)$ and DSC measurements). The low field part of the curve is rather steep showing that some fraction of the sample is in the ordered phase from the very start of the process. For this sample $\mu_{0} \Delta H=\mu_{0}\left(H_{80 \%}-H_{10 \%}\right)=3.9 \mathrm{~T}$ and $\mu_{0} H_{50 \%}=1$ $\mathrm{T}$. The conventionally sintered sample (red full squares in Fig. 3) shows a quite different behavior. In this case the transition advances through a series of step-like jumps separated by more gradual low change intervals. The transition appears to be clamped below an applied field of about $1.4 \mathrm{~T}$. The sample is entirely transformed at about $2.8 \mathrm{~T}$ after experiencing three abrupt transitions. In this case $\mu_{0} \Delta H=\mu_{0}\left(H_{80 \%}-H_{10 \%}\right)=1.9$ $\mathrm{T}$ is slightly overestimating the real field interval where the virgin transition take place that is more reasonably the interval between the field corresponding to the first and last jump, namely $\mu_{0} \Delta H_{\text {jump }}=1.4 \mathrm{~T}$. A similar behavior is observed on the SPS $50 \mathrm{MPa}$ sample (green hollow diamonds in Fig. 3). Here the transformation is smoother and is laying on a wider field interval, $\mu_{0} \Delta H=\mu_{0}\left(H_{80 \%}-H_{10 \%}\right)=3.8 \mathrm{~T}$ with $\mu_{0} H_{50 \%}=2.4$ T. Finally, the SPS $80 \mathrm{MPa}$ sample (blue full triangles in Fig. 3) does not achieve a field induced transformation in the field range from 0 to $4 \mathrm{~T}$. This seems in agreement with the fact that the first transition, in zero field DSC measurement is shifted by $8-9 \mathrm{~K}$ to a lower temperature (Fig. 2), so that in isothermal measurement we are trying to field induce the transformation at a temperature too far from the actual virgin $T_{c}$ to achieve it.

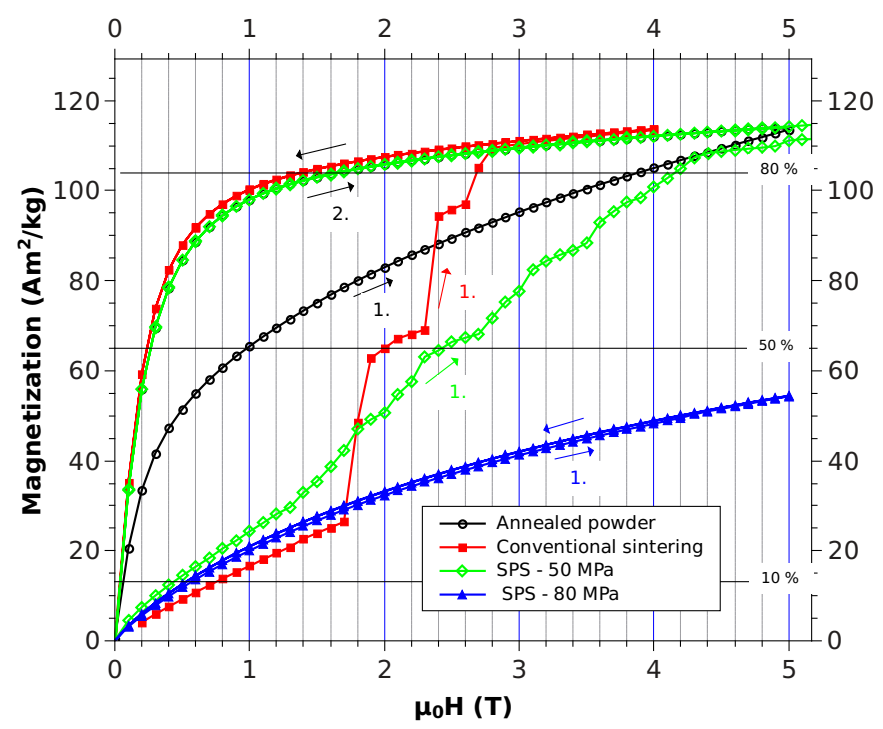

Figure 3: Isothermal magnetic measurements on the virgin samples at $250 \mathrm{~K}$.

\subsection{Hall probe imaging}

The isothermal field induced first (VE) and further transitions have been imaged using a scanning Hall probe microscope (SHPM) on the conventionally sintered sample. We followed the same protocol used to acquire isothermal magnetization curves with the PPMS. Virgin samples have been cooled to $250 \mathrm{~K}$ in zero magnetic field. Than images have been taken by increasing the field up to $4 \mathrm{~T}$ and decreasing it back to $0 \mathrm{~T}$ (Fig. 4). Hall probe images allow to visualize the jumps characterizing the first transition as sharp borderlines separating the FM regions bright in the images, from the dark PM ones (Fig. 4 sequence 1). The smooth decrease of $M$, mainly due to magnetization rotation, is measured when reducing $H$ to zero and is imaged as a gradual and uniform darkening of the sample (Fig. 4 sequence 2). Optical microscope images of the as-prepared sample (Fig. 5 left) and of the same sample after the first transition (Fig. 5 center) show the formation of cracks that correspond approximately in shape and position to 
the boundaries between FM and PM regions visualized with the Hall probe.

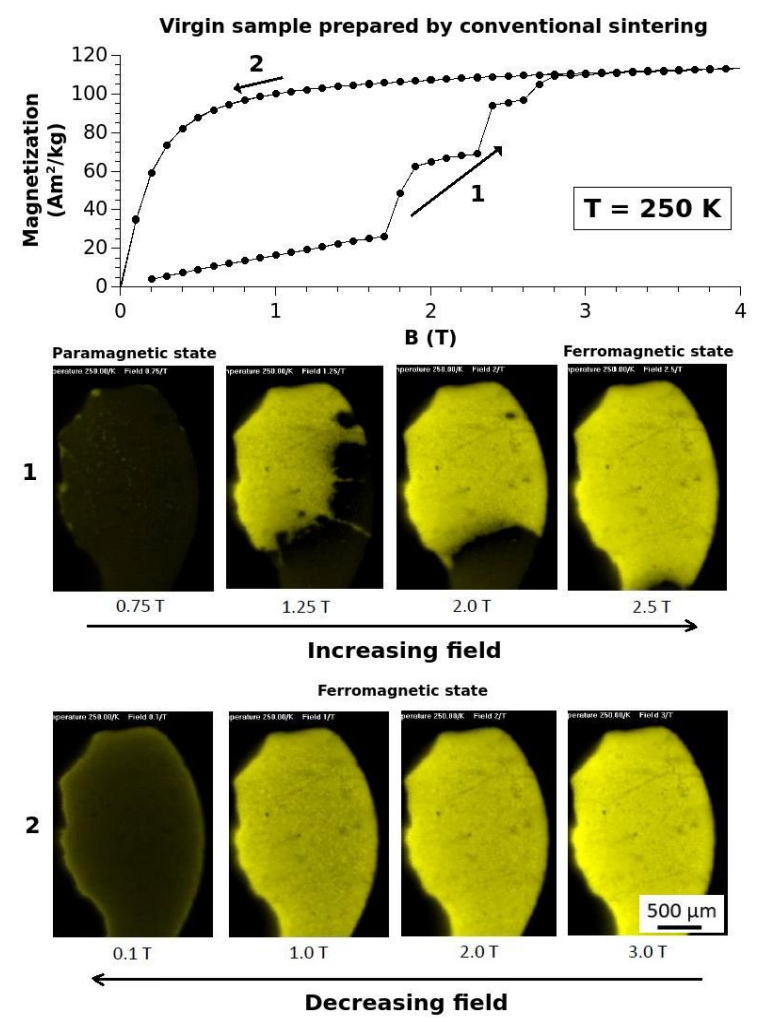

Figure 4: Isothermal magnetic measurements on the virgin sample prepared by conventional sintering at $250 \mathrm{~K}$ (top) and Hall probe imaging during measurement on the virgin sample prepared by conventional sintering at $250 \mathrm{~K}$ in increasing (center) and decreasing field (bottom). It is worth noting that the smooth darkening of the sample when the field decreases is entirely related to the rearrangement of $M$ and that the first image (the darkest one) shows a sample still ferromagnetic in its remanent state.

\section{Discussion and conclusions}

The results of this study present many similarities with a recent work on the virgin effect in LaFeSi [4] where the role of elastic energy due to inter-particle couplings is investigated.

In our case the main point of the discussion is the comparison between the VE phenomenologies we observed measuring the first transition of as-prepared samples under the action of a temperature change, in fixed field conditions (i.e. $M(T)$ curves and DSC data), and under the action of applied field change, in isothermal conditions (i.e. $M(H)$ curves and Hall probe images).

In the case of isofield magnetization measurements we do not have direct imaging of the process and, unfor-
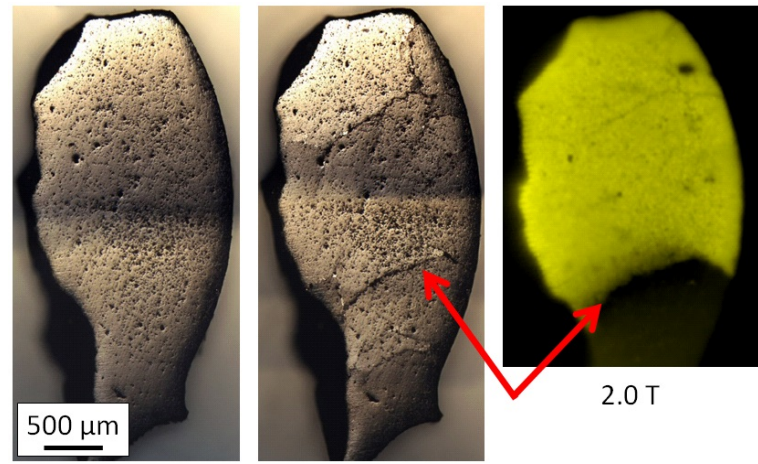

Figure 5: Optical image of sample prepared by conventional sintering. The as-prepared sample (left) and after the first transition (center), compared with a Hall image showing partial phase transition (right). Arrows are showing cracks corresponding approximately in shape and position to the boundaries between FM and PM regions.

tunately, the $M(T)$ curve resolution is not high enough to clearly show the jumps during the first transition. However, the resolution of the DSC was sufficient to capture the relevant data. The avalanche structure of the DSC VE measurements observed in bulk samples can be interpreted as associated with the formation of cracks similar in direct support of our observations using Hall probe imaging during the field induced VE. Moreover, the temperature interval where the transition takes place is larger for the powder sample $\left(\Delta T_{10 \%-80 \%}=46.9 \mathrm{~K}\right)$ than for the bulk ones $\left(\Delta T_{10 \%-80 \%}=13.4,30.3,32.1\right.$ $\mathrm{K}$ respectively for the conventionally sintered, SPS at $50 \mathrm{MPa}$ and SPS at $80 \mathrm{MPa}$ ). The result is quite similar when comparing $\Delta T_{D S C}$ (see Table 1). This behavior can be explained as associated with the elastic energy minimization during the transition. We assume that in this case elastic energy, due to the strain induced at the interface of coexistence between different phases, will be minimized by ordering huge regions and by constraining them to cross the transition at the same time. In $\mathrm{Fe}_{2} \mathrm{P}$ alloys, magnetic ordering is associated with a sharp increase of the $a$ lattice parameter of the hexagonal lattice [7]. We can understand that the grain boundaries between hexagonal crystals laying in the same plane but forming small angles between them, will be subject to rather significant strain during the transition. A low-angle grain boundary separating two crystals with a different orientation is commonly represented as a surface containing a sequence of dislocations. The virgin effect and the appearance of cracks could be associated with the rearrangement of arrays of dislocations associated with grain boundaries. In this frame dislocation rearrangement can be responsible for the virgin effect and, in the case where the grains are 
more closely mechanically interlocked, this will result in cracks [8]. As a general rule, freely mobile dislocations reduce the strength of materials [9]. From this point of view the difference in the behavior of the virgin effect in the bulk samples can be interpreted as due to different levels of dislocation mobility.

The dislocation mobility can be hindered by two mechanisms: pinning by defects and high dislocation density (inter-dislocation coupling). Defects distribution and dislocation density can both be at the origin of the difference in the sharpness of the first transition we observed in samples produced by different routes. Powder samples present a wide distribution of defects (also reflecting the distribution in grain sizes) whereas classical sintering induces a rather ordered structure with huge fractions of the sample mechanically coupled (this is the case where a long range elastic field can produce the sharp steps we observe in the field-induced transition). The case of SPS samples is different. SPS is a form of flash sintering where heating is fast and takes place from inside the sample due to the high current passing through the material. In this case we can expect to find a wider distribution of defects and a higher density of dislocations (the material is denser with smaller crystals and therefore with a higher total surface of inter-grain boundaries). This can explain why the 50 MPa SPS sample behaves in a way that is somewhere between the powder sample and the classically sintered one. In this frame we can also explain the seemingly anomalous behavior of the $80 \mathrm{MPa}$ SPS sample. Indeed, if we assume that increasing pressure can increase the dislocation density we can expect to obtain, in this case, a sample harder to deform (due to inter-dislocation coupling).

Of course further studies are needed to check this hypothesis. We shall perform an accurate structural characterization to estimate the effective surface of grain boundaries allowing a discussion about particles interlocking similar to the one in [4]. An interesting question is the comparison of our interpretation of the VE with those recently published in [3]. In that paper data from in-situ neutron diffraction experiments together with the recovery, by annealing, of the virgin behavior of samples have been interpreted as evidence of the existence of two, energetically degenerate, high temperature structural phases.

A comparison of our results with [3] can be interpreted in different ways. We can simply state that we are studying two essentially different effects: one, presented in this paper, is an extrinsic VE due to the polycrystalline nature of the samples and mainly related to the preparation route used producing the sample; the second, whereas in [3] the effect proposed is an intrinsic one, lead by the existence of two energetically degenerated high temperature structural phases in asprepared and in annealed samples. Alternatively, it is tempting to reconcile the two pictures in terms of the dislocation/boundary mobility concept. Indeed nucleation of a dislocation/defect configuration and the consequent hardening of a material can be obtained by annealing, and the dislocation density can be controlled tuning the annealing time and the quenching conditions. The whole process can be modeled with an activation approach similar to the one discussed in [3]. Moreover in-situ measurements showing local variations of lattice parameters and atomic positions can be interpreted as a signature of the presence of defects and dislocations and of the associated strain. Reconciliation of the two different approaches may only be possible after further work has been undertaken.

Here we show a first systematic study of the influence of particle size showing that the VE is drastically reduced for fine powders (i.e. particle size below 10 $\mu m)$. This suggests that there exists a critical particle size for the appearance of the virgin effect. In-situ structural characterizations of samples with different grain size will be needed to interpret this new data.

Notwithstanding the intrinsic vs. extrinsic origin of the VE, a final goal of great interest would be to relate the stable behavior of the transition (i.e. its sharpness and the presence of hysteresis) to microscopic features such as the defect distribution. This would facilitate the tailoring of the magnetocaloric properties by controlling the microstructure of materials using different preparation routes.

\section{Acknowledgement}

The research leading to these results has received funding from the European Union Seventh Framework Programme (FP7/2007-2013) under grant agreement $n^{\circ}$ 310748 (DRREAM).

\section{References}

[1] N. H. Dung, L. Zhang, Z. Q. Ou, E. Brück, From first-order magneto-elastic to magneto-structural transition in $(\mathrm{Mn}, \mathrm{Fe})_{1.95} \mathrm{P}_{0.50} \mathrm{Si}_{0.50}$ compounds, Applied Physics Letters 99 (9) (2011) 092511. doi:10.1063/1.3634016.

[2] E. Brck, N. Trung, Z. Ou, K. Buschow, Enhanced magnetocaloric effects and tunable thermal hysteresis in transition metal pnictides, Scripta Materialia 67 (6) (2012) 590 593, viewpoint Set No. 51: Magnetic Materials for Energy. doi:http://dx.doi.org/10.1016/j.scriptamat.2012.04.037. 
[3] X. F. Miao, L. Caron, Z. Gercsi, A. Daoud-Aladine, N. H. van Dijk, E. Brck, Thermal-history dependent magnetoelastic transition in (mn,fe)2(p,si), Applied Physics Letters 107 (4) (2015) -. doi:http://dx.doi.org/10.1063/1.4927285.

[4] A. Waske, L. Giebeler, B. Weise, A. Funk, M. Hinterstein, M. Herklotz, K. Skokov, S. Fhler, O. Gutfleisch, J. Eckert, Asymmetric first-order transition and interlocked particle state in magnetocaloric $\mathrm{La}(\mathrm{Fe}, \mathrm{Si}) 13$, physica status solidi (RRL) Rapid Research Letters 9 (2) (2015) 136-140. doi:10.1002/pssr.201409484.

[5] N. H. Dung, Z. Q. Ou, L. Caron, L. Zhang, D. T. C. Thanh, G. A. de Wijs, R. A. de Groot, K. H. J. Buschow, E. Brck, Mixed magnetism for refrigeration and energy conversion, Advanced Energy Materials 1 (6) (2011) 1215-1219. doi:10.1002/aenm.201100252.

[6] L. Caron, M. Hudl, V. Höglin, N. H. Dung, C. P. Gomez, M. Sahlberg, E. Brück, Y. Andersson, P. Nordblad, Magnetocrystalline anisotropy and the magnetocaloric effect in $\mathrm{Fe}_{2} \mathrm{P}$, Phys. Rev. B 88 (2013) 094440. doi:10.1103/PhysRevB.88.094440.

[7] N. H. Dung, L. Zhang, Z. Q. Ou, L. Zhao, L. van Eijck, A. M. Mulders, M. Avdeev, E. Suard, N. H. van Dijk, E. Brück, High/low-moment phase transition in hexagonal Mn-Fe-P-Si compounds, Phys. Rev. B 86 (2012) 045134. doi:10.1103/PhysRevB.86.045134.

[8] S. Zhou, A. Carlsson, R. Thomson, Dislocation nucleation and crack stability: lattice greens-function treatment of cracks in a model hexagonal lattice, Physical Review B 47 (13) (1993) 7710.

[9] L. D. Landau, E. Lifshitz, Theory of Elasticity, vol. 7, Vol. 3, Elsevier New York, 1986. 